\title{
Ultrasonic monitoring on glass fiber reinforced cement (GRC) bending test
}

\author{
V. Genovés ${ }^{1}$, J. Gosálbez ${ }^{2}$, R. Miralles ${ }^{2}$, L. Soriano ${ }^{1}$ \& J. Payá ${ }^{1}$ \\ ${ }^{1}$ Universitat Politècnica de València, ICITECH, Spain \\ ${ }^{2}$ Universitat Politècnica de València, ITEAM, Spain
}

\begin{abstract}
Glass fiber reinforced cement (GRC) is a composite material based on Portland cement and alkali resistant glass fibers. This composite has good mechanical properties including toughness and flexural strength. The characterization of this material is carried out by four points bending test. During this test, elastic and plastic behaviour are identified from load vs. deflection curves. The scope of the research is to monitor this bending test by means on ultrasonic pulses in order to detect changes on the material and to correlate them with mechanical behaviour. In this sense, pulse velocity, energy, attenuation and non-linearities of the ultrasonic received signals have been analysed. First changes in energy, attenuation and nonlinearities were detected during the change from elastic to plastic step and the first pulse velocity change were observed during the plastic step.

Keywords: glass fiber reinforced cement, ultrasound, attenuation, bending test, pulse velocity, non-linearities, energy.
\end{abstract}

\section{Introduction}

Glass fiber Reinforced Cement (GRC) is a composite made of Portland cement mortar with low water/cement $(\mathrm{w} / \mathrm{c})$ ratio with a large amount of paste in relation to the aggregate quantity. The addition of a high proportion of glass fibers to the mortar matrix ( $3 \%-5 \%$ by weight of mortar) improves the mechanical properties of the elements produced with this material reinforcement, especially the toughness, flexural strength and ductility. This composite has an important role in non-steel reinforced pre-cast concrete elements, such as sheets, panels and other slim shapes usually employed in building engineering and architecture, and also in cast-in-situ sprayed-on surfaces [1,2]. 
Recently, several researchers have focused on non-destructive concrete and mortar characterization in order to obtain more reliable information and new aspects of cementitious composites without damaging the specimen under test. During recent years, Non-Destructive Techniques (NDT) applied on concrete have been investigated, especially ultrasonics and acoustic spectroscopy, in order to obtain parameters related to the physical and mechanical properties of the material [3]. Some experimental studies have demonstrated that wave parameters such as the ultrasonic pulse velocity of P-waves are suitable to predict the dynamic elastic modulus of concrete and also $\mathrm{S}$-waves for dynamic shear modulus. These parameters are proportional to the elastic and shear moduli of concrete and also to its compressive strength $[4,5]$. Other studies based on ultrasound propagation indicate that wave attenuation can be measured for various frequencies, in order to define different cementitious materials, distinguishing the microstructure, porosity, and other characteristics in both hardened [6-9] and fresh states [10]. Some studies have shown interesting correlations between other properties of cementitious composites, such as their permeability or porosity, using ultrasonic parameters such as pulse velocity, signal attenuation, or reflection coefficients, by means of an specific test set-up.

Many studies on construction materials tests monitored by ultrasounds were published by researchers [11]. Research on metallic specimens are predominant because this material is used in other engineering fields. However, recent studies on reinforced concrete slabs bending tests with an ultrasonic monitoring were done successfully [12]. The scope of this paper is to monitor bending tests of GRC specimens in order to assess the changes on most important linear ultrasonic parameters (P-wave velocity, energy and attenuation) and non-linear ultrasonic behaviour.

\section{Experimental}

\subsection{Materials}

In this experiment the specimen dimension for bending test was chosen as $325 \times$ $50 \times 20 \mathrm{~mm}$.

The specimens were cut from $400 \times 400 \times 20 \mathrm{~mm}$ mother plates. From mother plate three specimens were obtained. Table 1 shows the proportions and information about the raw materials used to fabricate the GRC plate.

\subsection{Experimental layout}

An ultrasonic through-transmission setup was selected because it offers good penetration and good accuracy for velocity and attenuation estimation [6, 13-15]. The layout of the equipment is shown in Figure 1.

The transducers used were the K1SM (for transmission) and K1SC (for reception) from General Electric. Both are broadband transducers with a bandwidth centered at $1 \mathrm{MHz}$. 
Table 1: Used dosage for one GRC plate specimen.

\begin{tabular}{lll}
\hline Material & Type & Weight $[\mathrm{g}]$ \\
\hline Cement & CEM I 52.5 R & 7260 \\
Water & - & 2541 \\
Sand & Silica sand 0/2 & 4864 \\
Superplasticizer & polycarboxylate ether & 14 \\
Fiber & Glass AR 12 mm & 294 \\
\hline
\end{tabular}

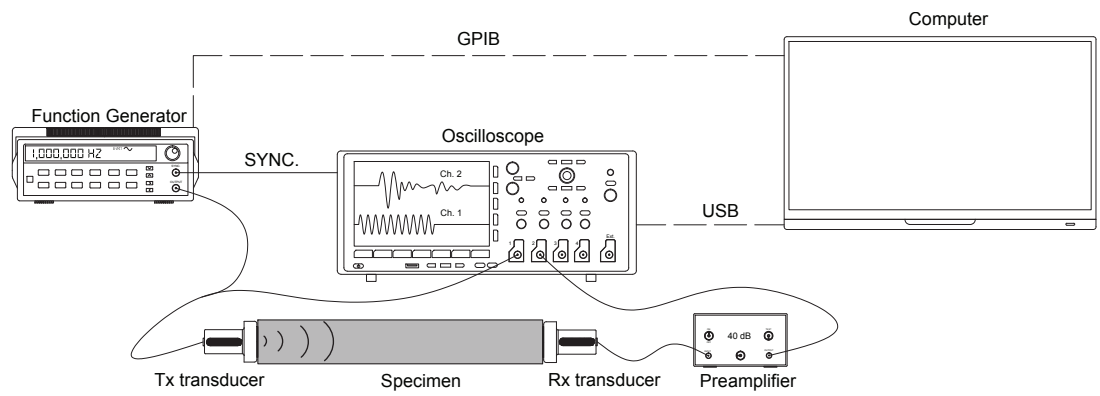

Figure 1: Ultrasonic equipment layout.

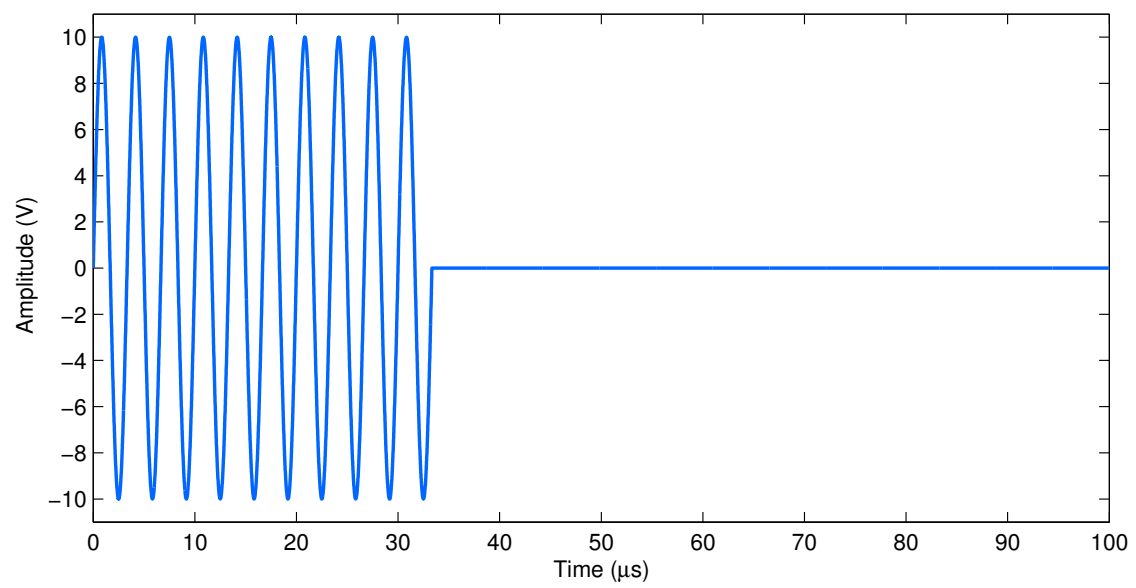

Figure 2: Tone burst signal: $f_{0}=300 \mathrm{kHz}, A_{t x}=10, N=10$.

GRC specimens were made according to the BS EN 1170-5 [16]. Instron universal testing machine (model 3382) was used for the mechanical test and the 
distance for the supports was $295 \mathrm{~mm}$ for passive supports and $98 \mathrm{~mm}$ for active ones. The displacement of the actuator was constant during the test $(1.2 \mathrm{~mm} / \mathrm{min})$.

The transmitter transducer was excited directly by a programmable signal generator (Agilent 33120A). The transmitted signal was a sinusoidal burst signal Eq. (1) and the selected values were: amplitude, $A_{t x}=10 \mathrm{~V}$, number of cycles, $N=10$, and fundamental frequency, $f_{0}=300 \mathrm{kHz}$ (Figure 2). The reception transducer was connected to a $40 \mathrm{~dB}$ preamplifier (Panametrics 5600B). The received and amplified ultrasonic signal was captured by a digital oscilloscope (Tektronix DPO3014) with a sampling frequency of $25 \mathrm{MHz}$. Finally, a laptop was used to control the signal generator and to acquire and store the digitized signals by the oscilloscope.

$$
x_{t x}(t)=\left\{\begin{array}{l}
A_{t x} \sin \left(2 \pi f_{0} t\right), \quad 0<t<N T_{0} \\
0, \quad \text { otherwise }
\end{array}\right.
$$

In Figure 3 the experimental layout of simultaneous mechanical test and ultrasonic continuous data acquisition system is shown. The plate rests on the passive supports and four metallic angles were attached to it. These elements serve to hold the elastic bands which keep the pressure of the transducers on the faces of the plate. This system is similar to that other authors used previously, in which rubber bands keep the appropriate coupling between transducers and specimen [17]. To ensure proper coupling between transducer and specimen faces, water-based gel was used.

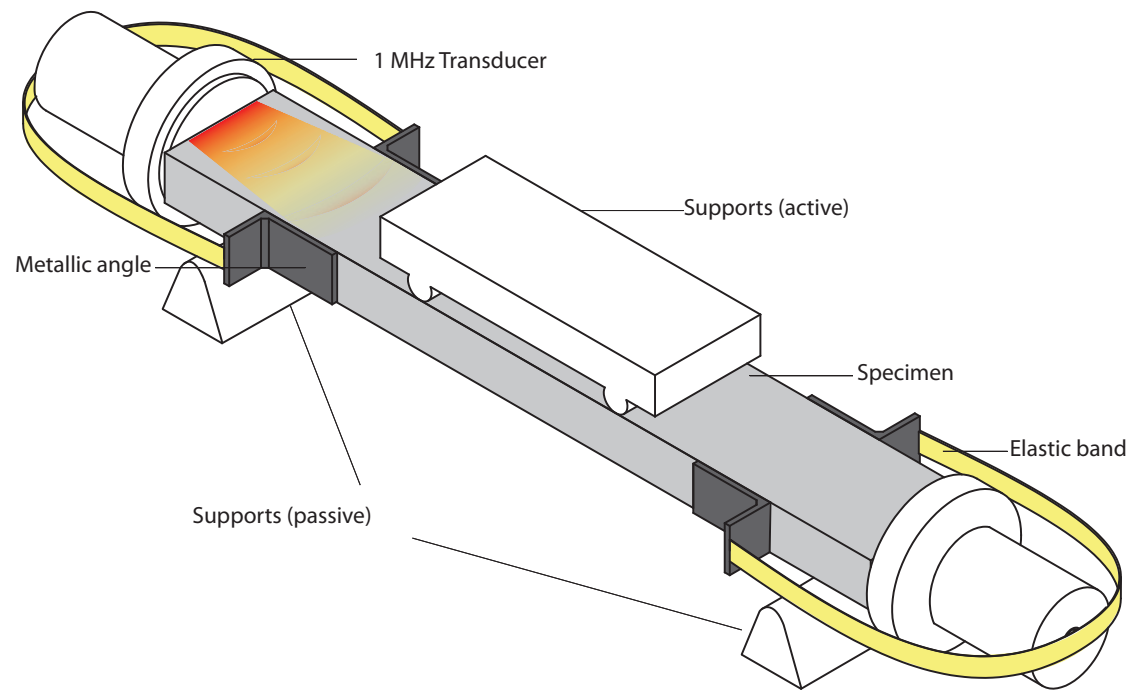

Figure 3: Experimental layout. 


\subsection{Monitorized ultrasound parameters}

The estimated ultrasound parameters from received ultrasonic signal are: p-wave velocity, received energy, attenuation of the material and non-linear behaviour. These parameters will depend on the time of the assay $(t)$.

- P-wave velocity $\left(v_{p}(t)[\mathrm{m} / \mathrm{s}]\right)$ is obtained as the ratio between the length of the specimen, $d_{m a t}$, and the signal time arrival, $t_{a}(t)$, Eq. (2). The $t_{a}$ was estimated as the time when the received signal level exceeds $50 \%$ of the noise level.

$$
v_{p}(t)=\frac{d_{m a t}}{t_{a}(t)}
$$

- Received energy, $E_{r x}(t)[\mathrm{J}]$, is the energy of the received signal measured by the oscilloscope. The $x$ is the amplitude of the signal at each instant of time $\tau$. Its value is obtained from Eq. (3).

$$
E_{r x}(t)[\mathrm{J}]=\int_{0}^{\infty} x_{r x, t}^{2}(\tau) d \tau
$$

- Attenuation of the material, $\alpha_{m a t}(t)[\mathrm{dB} / \mathrm{cm}]$, is obtained as the difference between transmitted energy, $E_{t x}[\mathrm{~dB}]$ and the received energy, $E_{r x}(t)[\mathrm{dB}]$, plus attenuation due to the equipment, $\alpha_{\text {equip }}[\mathrm{dB}]$, divided by the total length of the specimen, $d_{\text {mat }}[\mathrm{cm}]$, Eq. (4). Taking into account that analytical expression and specific parameters of the transmitted signal are known, Eq. (1), $E_{t x}$ can be obtained theoretically using Eq. (5). This value, $1.7 \mathrm{~mJ}$, is constant through the test. Meanwhile $E_{r x}(t)$ is obtained from Eq. (3). The $\alpha_{\text {equip }}=-5 \mathrm{~dB}$ is the attenuation associated to the measurement equipment (transducers, amplifier, cables, acquisition module, ...) and, it is independent of the tested material and it is constant through the test although a calibration process was required.

$$
\begin{gathered}
\alpha_{\text {mat }}(t)[\mathrm{dB} / \mathrm{cm}]=\frac{E_{t x}-E_{r x}(t)+\alpha_{\text {equip }}}{d_{\text {mat }}} \\
E_{t x}[\mathrm{~J}]=A_{t x}^{2} N \frac{1}{2 f_{0}}
\end{gathered}
$$

- $\boldsymbol{\beta}_{3}(\boldsymbol{t})$ was selected as non-linear parameter. It is defined as the ratio between the amplitude of the third harmonic $\left(A_{3}\right)$ and the fundamental harmonic $\left(A_{1}\right)$ following Eq. 6 and it is very sensitive to the formation of cracks. Therefore, the variation of this parameter respect to its initial value will be represented as $\beta_{3}^{\%}(t)$, according to Eq. (7), where $\beta_{3}^{\%}(0)$ is the non-linear parameter before beginning the loading $(t=0)$.

$$
\begin{gathered}
\beta_{3}(t)=\frac{A_{3}(t)}{A_{1}^{3}(t)} \\
\beta_{3}^{\%}(t)=100 \frac{\beta_{3}(t)}{\beta_{3}(0)}
\end{gathered}
$$




\section{Results and discussion}

Mechanical parameters of each plate were obtained from bending tests (Table 2).

Table 2: Mechanical parameters extracted from bending test.

\begin{tabular}{llll}
\hline Specimen & Toughness $[\mathrm{Nmm}]$ & $\mathrm{Ec}_{\text {flex }}[\mathrm{GPa}]$ & $\sigma_{\max }[\mathrm{MPa}]$ \\
\hline A & 595 & 18.2 & 10.3 \\
B & 438 & 17.3 & 9.5 \\
C & 794 & 23.5 & 12.9 \\
\hline
\end{tabular}

After completion of the tests, ultrasound parameters and load vs. extension curves were plotted on the same figures. The time $x$-axis was used because the active supports velocity was constant throughout the test as described in the experimental section, thus the understanding of the graphs is improved.

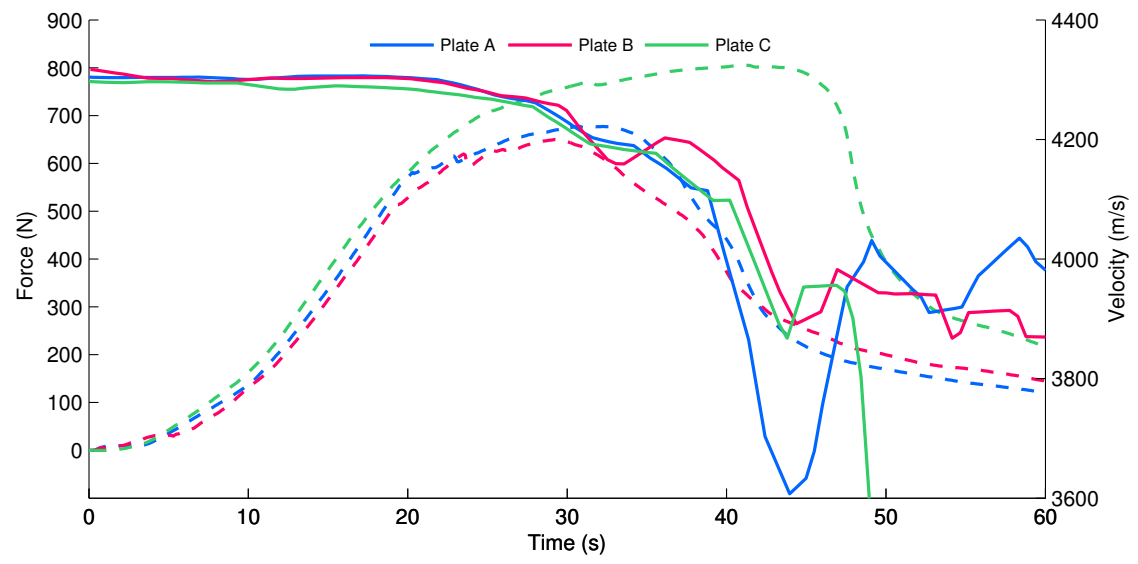

Figure 4: P-wave velocity and Force vs. Time.

In Figure 4, P-wave velocity was plotted for each specimen. It can be observed that the wave velocity $\left(v_{p}\right)$ does not change during the elastic material behaviour $(t=0-25 \mathrm{~s})$. When the material comes in plastic stage $(t=25-35 \mathrm{~s})$, pwave velocity changes to lower values due to the micro-cracking process. This microcracking produce a diminution in the Young modulus. When the materials was very damaged $(t<40 \mathrm{~s}) v_{p}$ values shown a non-defined trend because the large size of cracks.

Velocity parameter fluctuates after 40 seconds of testing because the signal amplitude is too low to determine correctly the time of flight of the ultrasonic 


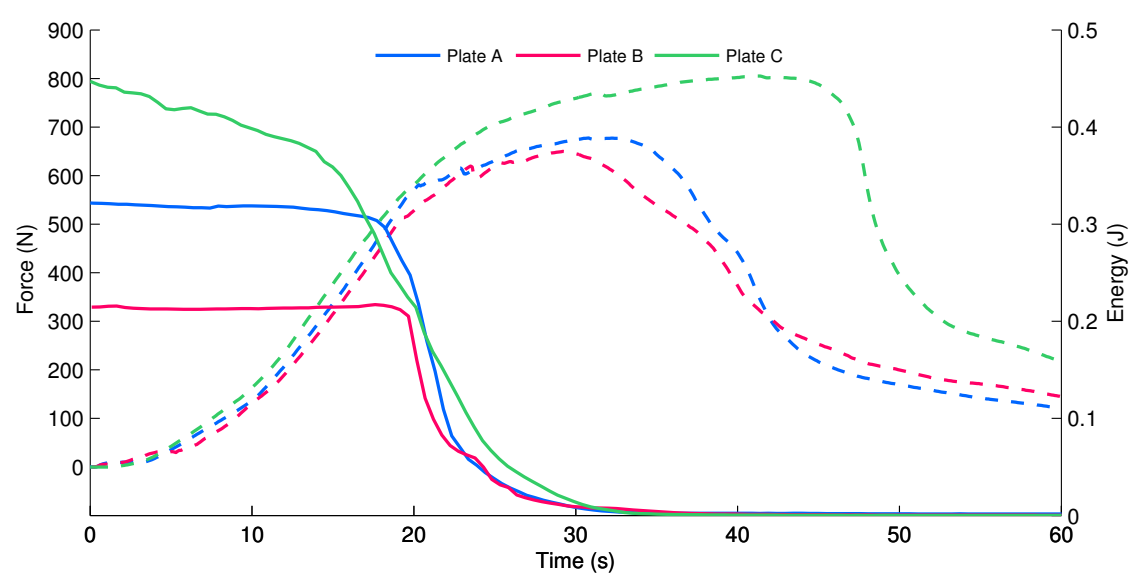

Figure 5: Received energy and Force vs. Time.

signal. In Figure 5, received energy $\left(\mathrm{E}_{r x}\right)$ was plotted for each specimen. It can be observed that the energy is constant during the elastic phase of the curve.

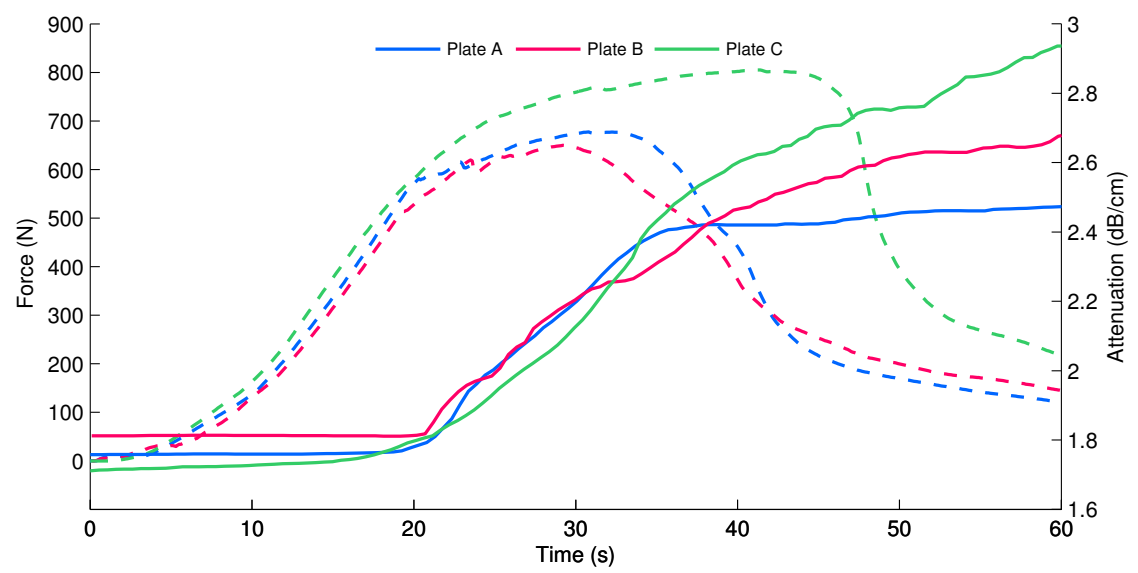

Figure 6: Material attenuation and Force vs. Time.

However, when the first microcracks appeared (change from elastic to plastic phase) the $\mathrm{E}_{r x}$ parameter drastically drops to very low values. Thus, after the plastic step, the value was negligible. This behaviour means that the energy transmission strongly depends on the formation of the first microcracks during the test. When the material comes in plastic stage, energy decays quickly to zero due to the cracks and subsequent section loss. 
Figure 6 shows the attenuation $\left(\alpha_{m a t}\right)$ of the material during the bending test. This parameter is inversely related to received energy $\left(\mathrm{E}_{r x}\right)$, therefore it has similar trends. The $\alpha_{m a t}$ is being constant at the beginning of the assay and reaching higher values when the plastic stage starts. Non-linear parameter $\left(\beta_{3}^{\%}(t)\right)$ curves were plotted in Figure 7 . This parameter shows clearly the instant when the curve in elastic stage turns on plastic behaviour due to the micro-cracking (and subsequent macro-cracking and section loss), beginning with a slight increment for few seconds (between 15 and 20 seconds testing time) and high increment when plastic stage begins indicating greater damage.

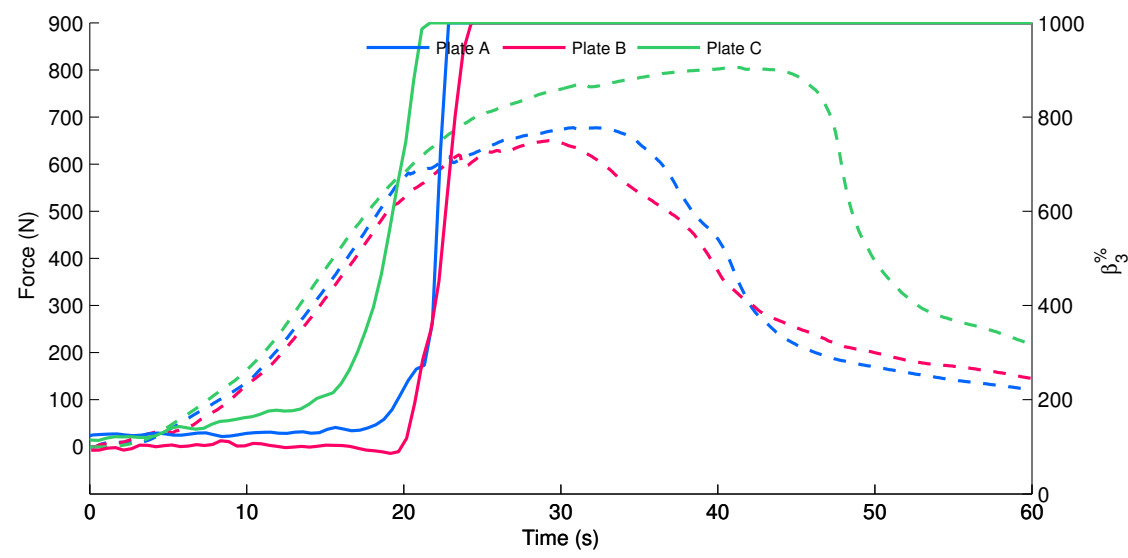

Figure 7: Non-linear parameter and Force vs. Time.

As can be observed, both mechanical and ultrasonic parameters behaviours were very similar among three specimens. In Figure 8 all parameters for one specimen (plate B) are plotted in order too see directly differences among them during the test. Mechanical and ultrasonic parameters were normalized, thus all values were in the 0-1 range. All wave parameters are sensitive to the changes of the material when the mechanical behaviour of the material turns from elastic to plastic stage but $\beta_{3}^{\%}(t)$ has a sudden change on early plastic stage, marking the beginning of the plastic deformations.

\section{Conclusions}

The bending test process of glass fiber reinforced cement (GRC) has been successfully monitored by means of the study of ultrasonic pulses. Loading (constant displacement rate) and ultrasonic parameters were compared in the time scale. Elastic deformation step in the mechanical test does not produce significative changes in pulse velocity, energy, attenuation and non-linearities of the ultrasonic received signals. In the beginning of the plastic step in the mechanical test, significant changes in energy, attenuation and non-linearities has 


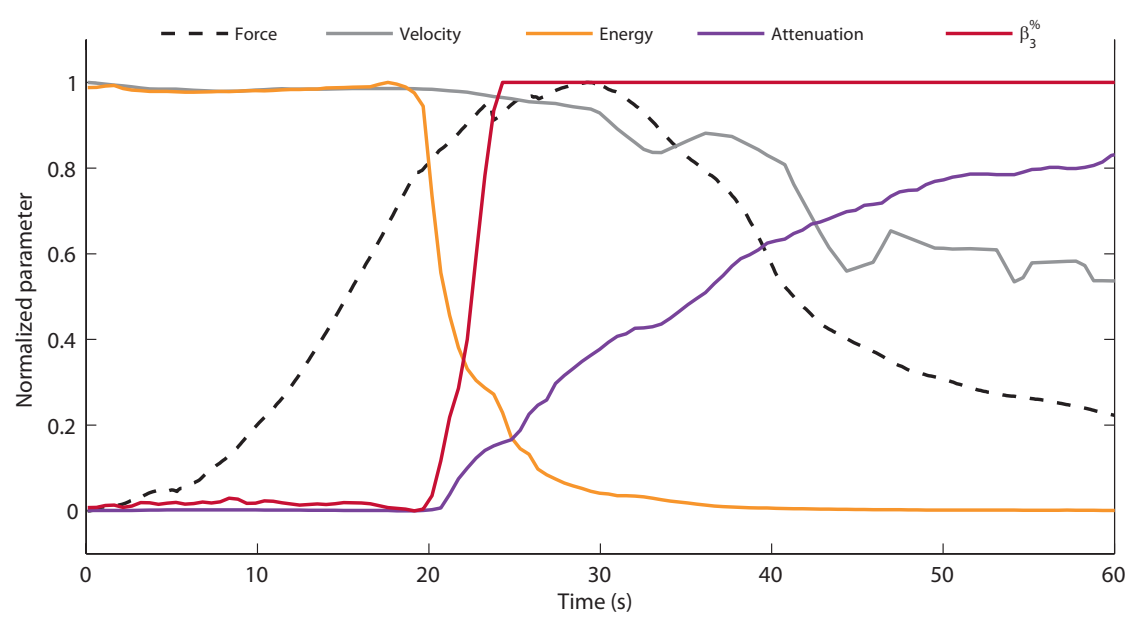

Figure 8: Normalized parameters vs. Time.

been identified. However, the change in the velocity pulse was very less important. The change in the ratio of the fundamental harmonic $\left(\mathrm{A}_{1}\right)$ and the third harmonic $\left(\mathrm{A}_{3}\right)$ was the highest found, being thus this parameter (non-linearity) the most relevant for detecting the plastic step. In this step, microcracking of the matrix has been produced because the mechanical loading, and this alteration in the matrix activates the generation of non-linearities.

\section{References}

[1] Majumdar, A.J. \& Ryder, J., Glass fibre reinforcement of cement products. Garston, UK: Building Research Station, 1968.

[2] Bentur, A., Fibre reinforced cementitious composites. Taylor \& Francis, p. 625, 1990.

[3] V.M. Maholtra, N.C., Non destructive testing on concrete, volume 1. CRC Press, 2004.

[4] Bogas, J.A., Gomes, M.G. \& Gomes, A., Compressive strength evaluation of structural lightweight concrete by non-destructive ultrasonic pulse velocity method. Ultrasonics, 53(5), pp. 962-972, 2013.

[5] Jain, A., Kathuria, A., Kumar, A., Verma, Y. \& Murari, K., Combined use of non-destructive tests for assessment of strength of concrete in structure. Procedia Engineering, 54(0), pp. 241-251, 2013.

[6] Philippidis, T.P. \& Aggelis, D.G., Experimental study of wave dispersion and attenuation in concrete. Ultrasonics, 43(7), pp. 584-95, 2005.

[7] Popovics, S., Rose, J.L. \& Popovics, J.S., The behaviour of ultrasonic pulses in concrete. Cement and Concrete Research, 20(2), pp. 259-270, 1990. 
[8] Vergara, L., Gosálbez, J., Fuente, J., Miralles, R. \& Bosch, I., Measurement of cement porosity by centroid frequency profiles of ultrasonic grain noise. Signal Processing, 84(12), pp. 2315-2324, 2004.

[9] Vergara, L., Miralles, R., Gosálbez, J., Juanes, F., Ullate, L., Anaya, J., Hernández, M. \& Izquierdo, M., NDE ultrasonic methods to characterise the porosity of mortar. NDT \& E International, 34(8), pp. 557-562, 2001.

[10] Aggelis, D. \& Philippidis, T., Ultrasonic wave dispersion and attenuation in fresh mortar. NDT \& E International, 37(8), pp. 617-631, 2004.

[11] Ogi, H., Hamaguchi, T. \& Hirao, M., In-situ monitoring of ultrasonic attenuation during rotating bending fatigue of carbon steel with electromagnetic acoustic resonance. Journal of Alloys and Compounds, 310(1-2), pp. 436439, 2000.

[12] Moradi-Marani, F., Rivard, P., Lamarche, C. \& Kodjo, S., Evaluating the damage in reinforced concrete slabs under bending test with the energy of ultrasonic waves. Construction and Building Materials, 73, pp. 663-673, 2014.

[13] Krautkrämer, J. \& Krautkrämer, H., Ultrasonic testing of materials. Springer, 1983.

[14] Gaydecki, P. \& Burdekin, F., The propagation and attenuation of mediumfrequency ultrasonic waves in concrete: a signal analytical approach. Measurement Science, 126, p. 400, 1992.

[15] Molero, M., Segura, I., Aparicio, S., Hernández, M. \& Izquierdo, M., On the measurement of frequency-dependent ultrasonic attenuation in strongly heterogeneous materials. Ultrasonics, 50(8), pp. 824-828, 2010.

[16] British Standard, Precast concrete products - test method for glass-fibre reinforced cement - part 5. measuring bending strength, 'complete bending test' method, 1998.

[17] Shah, A. \& Ribakov, Y., Non-linear ultrasonic evaluation of damaged concrete based on higher order harmonic generation. Materials \& Design, 30(10), pp. 4095-4102, 2009. 